\title{
Finite Element Modeling of Thermopiezomagnetic Smart Structures
}

\author{
M. Sunar, ${ }^{*}$ Ahmed Z. Al-Garni, ${ }^{\dagger}$ M. H. Ali, ${ }^{\ddagger}$ and R. Kahraman ${ }^{\S}$ \\ King Fahd University of Petroleum and Minerals, Dhahran 31261, Saudi Arabia
}

\begin{abstract}
Linear constitutive equations of a thermopiezomagnetic medium involving mechanical, electrical, magnetic, and thermal fields are presented with the aid of a thermodynamic potential. A thermopiezomagnetic medium can be formed by bonding together a piezoelectric and magnetostrictive composite. Two energy functionals are defined. It is shown via Hamilton's principle that these functionals yield the equations of motion for the mechanical field, Maxwell's equilibrium equations for the electrical and magnetic fields, and the generalized heat equation for the thermal field. Finite element equations for the thermopiezomagnetic media are obtained by using the linear constitutive equations in Hamilton's principle together with the finite element approximations. The finite element equations are utilized on an example two-layer smart structure, which consists of a piezoceramic (barium titanate) layer at the bottom and a magnetoceramic (cobalt ferrite) layer at the top. An electrostatic field applied to the piezoceramic layer causes strain in the structure. This strain then produces magnetic field in the magnetoceramic layer.
\end{abstract}

\section{Nomenclature}

$\begin{array}{ll}\boldsymbol{A} & =\text { vector of magnetic potential } \\ \boldsymbol{B} & =\text { vector of magnetic flux density } \\ b & =\text { matrix of electromagnetic coefficients } \\ c & =\text { matrix of elastic stiffness coefficients } \\ \boldsymbol{D} & =\text { vector of electrical displacement } \\ \boldsymbol{E} & =\text { vector of electrical field intensity } \\ \boldsymbol{e} & =\text { matrix of piezoelectric coefficients } \\ \boldsymbol{G} & =\text { thermodynamic potential } \\ \boldsymbol{H} & =\text { vector of magnetic field intensity } \\ \boldsymbol{h} & =\text { vector of external (applied) heat flux } \\ \boldsymbol{I} & =\text { area moment of inertia about the neutral axis } \\ \boldsymbol{J} & =\text { vector of volume current density } \\ K & =\text { matrix of heat conduction coefficients } \\ \boldsymbol{\ell} & =\text { matrix of piezomagnetic stress coefficients } \\ \boldsymbol{n} & =\text { vector of surface normal } \\ \boldsymbol{P} & =\text { vector of pyroelectric coefficients } \\ \boldsymbol{P}_{b} & =\text { vector of body forces } \\ \boldsymbol{P}_{c} & =\text { vector of concentrated forces } \\ \boldsymbol{P}_{s} & =\text { vector of surface forces } \\ \boldsymbol{q} & =\text { vector of heat flux } \\ \boldsymbol{r} & =\text { vector of thermomagnetic coefficients } \\ \boldsymbol{S} & =\text { strain vector } \\ \boldsymbol{T} & =\text { stress vector } \\ \boldsymbol{u} & =\text { displacement vector } \\ W & =\text { heat source density } \\ \alpha & =\text { entropy constitutive coefficient } \\ \varepsilon & =\text { matrix of dielectric coefficients } \\ \eta & =\text { entropy density } \\ \Theta & =\text { absolute temperature } \\ \Theta_{0} & =\text { reference temperature } \\ \theta & =\text { small temperature variation } \\ \lambda & =\text { vector of thermal stress coefficients } \\ \mu & =\text { matrix of permeability coefficients } \\ \rho & =\text { mass density } \\ & \end{array}$

Received 30 September 1999; revision received 17 May 2001; accepted for publication 17 July 2001. Copyright (C) 2002 by the authors. Published by the American Institute of Aeronautics and Astronautics, Inc., with permission. Copies of this paper may be made for personal or internal use, on condition that the copier pay the $\$ 10.00$ per-copy fee to the Copyright Clearance Center, Inc., 222 Rosewood Drive, Danvers, MA 01923; include the code 0001-1452/02 \$10.00 in correspondence with the CCC.

*Associate Professor, Mechanical Engineering Department.

${ }^{\dagger}$ Professor, Mechanical Engineering Department. Senior Member AIAA.

${ }^{*}$ Research Assistant, Mechanical Engineering Department.

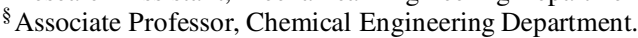

$$
\begin{aligned}
& \rho_{v}=\text { volume charge density } \\
& \sigma=\text { surface charge } \\
& \phi=\text { electrical potential }
\end{aligned}
$$

\section{Introduction}

$\mathbf{M}$ ECHANICAL, electrical, magnetic, and thermal fields coexist in some physical phenomena when the host medium is suitable. The general problem involving these fields is a complex problem caused by many issues that must be addressed for the solution. In most studies the interaction of only two or three fields are considered. The constitutiverelations among various fields are often linearized assuming that the fields are in quasi-static equilibrium at a given instant.

When the magnetic field is ignored, the interactions of remaining fields lead to the phenomenon of thermopiezoelectricity. If only mechanical and electrical fields are considered, the governing equations reduce to those of piezoelectricity. The piezoelectricity was discovered by the Curie brothers in $1880 .{ }^{1}$ The piezoelectric transducers have been used in numerous applications over a century. Although the piezoelectricity has a long history, its application to system control has happened only in recent years, practically in the late 1980s and in the 1990s. The past decade has witnessed a remarkable increase in the research of piezoelectricity pertaining to activities in system sensing and control, ${ }^{2,3}$ The recent review papers by Crawley, ${ }^{4}$ Newnham, ${ }^{5}$ and Sunar and Rao $^{6}$ report the increasing trend of research in piezoelectrical sensing and actuation. Piezoelectric polymer polyvinylidenefluoride (PVDF) and piezoceramics such as barium titanate $\left(\mathrm{BaTiO}_{3}\right)$ and lead zirconate titanate (PZT) are some piezoelectric materials used in various applications.

The governing equations of thermopiezoelectricity were first derived by Mindlin. ${ }^{7}$ A system of two-dimensional equations for high-frequency motions of thermopiezoelectric crystal plates was obtained by Mindlin. ${ }^{8}$ Some general theorems of thermopiezoelectricity were given by Nowacki. ${ }^{9}$ The application of thermopiezoelectricity to the control of adaptive structures has been carried out in recent years. Analysis of distributed thermopiezoelectricsensors and actuators for intelligent structures was performed by Rao and Sunar. ${ }^{10}$ The quasi-static equations of thermopiezoelectricity were used to develop a finite element formulation for thermopiezoelectric media. The formulation was utilized in studying thermal effects in intelligent structures for vibration control. Thermopiezoelectric actuator placement problem for cantilever-beamlike structures was considered by Sunar and Rao. ${ }^{11}$ The precision control by piezoelectric systems operation under thermal influences was presented by Tzou and Ye. ${ }^{12}$ Distributed control of piezoelectric laminates subjected to a steady-state temperature field was investigated. The 
thermopiezoelectric behavior of laminated plates was studied by Tauchert. ${ }^{13}$

In general, the phenomenon of magnetostriction is defined as the relation between mechanical and magnetic fields in a body. This phenomenon was discoveredby Joule in $1842 .{ }^{14}$ Some magnetostrictive ceramics can be listed as cobalt ferrite $\left(\mathrm{CoFe}_{2} \mathrm{O}_{4}\right), \mathrm{Ni}$, Alfenol, and Terfenol-D. Thin films with high magnetostrictionare very attractive for active mechanical actuators. The modeling of magnetostrictive thin films and application to a micromembrane was presented by Body et al. ${ }^{14}$

The piezoelectric and magnetostrictive layers can be bonded together to form composites that exhibit magnetoelectric effect. As a result of this effect, an electrical signal is obtained from the piezoelectric layer as a result of the application of a magnetic field to the magnetostrictive layer. Conversely, the magnetostrictive layer can be magnetized because of the application of an electrical field to the piezoelectric layer. These composites can be used as sensors and actuators for system control. A magnetoelectric coefficient was defined for these composites by Harshe et al. ${ }^{15}$ as the coefficient relating the electrical field to the magnetic field at open circuit conditions. Various piezoelectric/magnetostrictive composite combinations were formed by Avellaneda and Harshe ${ }^{16}$ to calculate the magnetoelectric coefficient and parameters that characterize the efficiency of energy conversion among the layers. A hybrid device composed of a magnetostrictivefilm on a piezoelectric substrate was described by Arai et al. ${ }^{17}$ The changes in the properties of the magnetostrictive film were observed as a result of electrical field applications on the piezoelectric substrate. The performance of a sensor consisting of a piezoelectric plate and magnetostrictive amorphous ribbon was studied by Prieto et al. ${ }^{18}$ The effect of the anisotropy induced by the piezoelectric plate on the ribbon's performance was investigated.

It appears from the literature survey that numerous papers have been published in the areas of piezoelectricity and magnetostriction in recent years. However, many research studies have been confined to either of these areas. Therefore, there is a need to present the equations of piezoelectricity and magnetostriction together, which can coexist in a thermopiezomagneticmedium. This medium can be formed to make use of both the piezoelectric and magnetostrictive effects for sensing and actuation.

In this paper the constitutive equations are obtained for a thermopiezomagnetic medium where mechanical, electrical, magnetic, and thermal fields interact with each other. These relations are general equations that can be used to describe the phenomena of linear thermopiezoelectricity, piezoelectricity, and magnetostriction, and their interactions. The constitutive equations are utilized in Hamilton's principle with the aid of two energy functionals. It is shown that the Hamilton's principle yields the equations of motion, Maxwell's equilibrium equations and the generalized heat equation for the mechanical, electrical and magnetic, and thermal fields, respectively. The finite element method is then applied to obtain the approximate linear equations that can be used to study the dynamic behavior of a thermopiezomagnetic medium in a comprehensive manner. A numerical example is given to illustrate the interaction of piezoelectricity and magnetostriction on a composite composed of $\mathrm{BaTiO}_{3}$ and $\mathrm{CoFe}_{2} \mathrm{O}_{4}$ layers.

\section{Constitutive Equations of Thermopiezomagnetism}

A thermodynamic potential $G$ is defined such that

$$
G=G(\boldsymbol{S}, \boldsymbol{E}, \boldsymbol{B}, \theta)
$$

The total differential of $G$ is

$$
\mathrm{d} G=\left(\frac{\partial G}{\partial \boldsymbol{S}}\right) \cdot \mathrm{d} \boldsymbol{S}+\left(\frac{\partial G}{\partial \boldsymbol{E}}\right) \cdot \mathrm{d} \boldsymbol{E}+\left(\frac{\partial G}{\partial \boldsymbol{B}}\right) \cdot \mathrm{d} \boldsymbol{B}+\left(\frac{\partial G}{\partial \theta}\right) \cdot \mathrm{d} \theta
$$

where the bold partial derivatives indicate the derivatives with respect to appropriate components of the correspondingvector and the subscripts are omitted for brevity. This scheme is followed throughout the paper. The partial derivatives in Eq. (2) are

$$
\begin{aligned}
\left(\frac{\partial G}{\partial \boldsymbol{S}}\right) & =\boldsymbol{T}, & \left(\frac{\partial G}{\partial \boldsymbol{E}}\right) & =-\boldsymbol{D} \\
\left(\frac{\partial G}{\partial \boldsymbol{B}}\right) & =\boldsymbol{H}, & \left(\frac{\partial G}{\partial \theta}\right) & =-\eta
\end{aligned}
$$

It is shown in the next section that the definition of these partial derivatives as in Eq. (3) results in the equations of motion for the mechanical field, Maxwell's equilibriumequations for the electrical and magnetic fields, and generalized heat equation for the thermal field. The total differentials of $\boldsymbol{T}, \boldsymbol{D}, \boldsymbol{H}$, and $\eta$ are as follows:

$$
\begin{gathered}
\mathrm{d} \boldsymbol{T}=\left(\frac{\partial \boldsymbol{T}}{\partial \boldsymbol{S}}\right) \mathrm{d} \boldsymbol{S}+\left(\frac{\partial \boldsymbol{T}}{\partial \boldsymbol{E}}\right) \mathrm{d} \boldsymbol{E}+\left(\frac{\partial \boldsymbol{T}}{\partial \boldsymbol{B}}\right) \mathrm{d} \boldsymbol{B}+\left(\frac{\partial \boldsymbol{T}}{\partial \theta}\right) \mathrm{d} \theta \\
\mathrm{d} \boldsymbol{D}=\left(\frac{\partial \boldsymbol{D}}{\partial \boldsymbol{S}}\right) \mathrm{d} \boldsymbol{S}+\left(\frac{\partial \boldsymbol{D}}{\partial \boldsymbol{E}}\right) \mathrm{d} \boldsymbol{E}+\left(\frac{\partial \boldsymbol{D}}{\partial \boldsymbol{B}}\right) \mathrm{d} \boldsymbol{B}+\left(\frac{\partial \boldsymbol{D}}{\partial \theta}\right) \mathrm{d} \theta \\
\mathrm{d} \boldsymbol{H}=\left(\frac{\partial \boldsymbol{H}}{\partial \boldsymbol{S}}\right) \mathrm{d} \boldsymbol{S}+\left(\frac{\partial \boldsymbol{H}}{\partial \boldsymbol{E}}\right) \mathrm{d} \boldsymbol{E}+\left(\frac{\partial \boldsymbol{H}}{\partial \boldsymbol{B}}\right) \mathrm{d} \boldsymbol{B}+\left(\frac{\partial \boldsymbol{H}}{\partial \theta}\right) \mathrm{d} \theta \\
\mathrm{d} \eta=\left(\frac{\partial \eta}{\partial \boldsymbol{S}}\right) \mathrm{d} \boldsymbol{S}+\left(\frac{\partial \eta}{\partial \boldsymbol{E}}\right) \mathrm{d} \boldsymbol{E}+\left(\frac{\partial \eta}{\partial \boldsymbol{B}}\right) \mathrm{d} \boldsymbol{B}+\left(\frac{\partial \eta}{\partial \theta}\right) \mathrm{d} \theta
\end{gathered}
$$

where the partial derivatives are defined as

$$
\frac{\partial \boldsymbol{T}}{\partial \boldsymbol{S}}=c, \quad \frac{\partial \boldsymbol{T}}{\partial \boldsymbol{E}}=-e, \quad \frac{\partial \boldsymbol{T}}{\partial \boldsymbol{B}}=-\ell, \quad \frac{\partial \boldsymbol{T}}{\partial \theta}=-\lambda
$$

The negative signs are from the negative stresses that must be applied in order to keep the material in the constant strain state. ${ }^{19}$ In view of Eqs. (3-5),

$$
\begin{gathered}
\frac{\partial^{2} G}{\partial \boldsymbol{S} \partial \boldsymbol{E}}=\frac{\partial \boldsymbol{T}}{\partial \boldsymbol{E}}=-\frac{\partial \boldsymbol{D}}{\partial \boldsymbol{S}}=-e, \quad \frac{\partial^{2} G}{\partial \boldsymbol{S} \partial \boldsymbol{B}}=\frac{\partial \boldsymbol{T}}{\partial \boldsymbol{B}}=\frac{\partial \boldsymbol{H}}{\partial \boldsymbol{S}}=-\ell \\
\frac{\partial^{2} G}{\partial \boldsymbol{S} \partial \theta}=\frac{\partial \boldsymbol{T}}{\partial \theta}=-\frac{\partial \eta}{\partial \boldsymbol{S}}=-\lambda
\end{gathered}
$$

Other partial derivatives in Eq. (4) are defined as

$$
\begin{aligned}
\frac{\partial \boldsymbol{D}}{\partial \boldsymbol{E}}=\varepsilon, & \frac{\partial \boldsymbol{D}}{\partial \boldsymbol{B}}=b, & \frac{\partial \boldsymbol{D}}{\partial \theta}=\boldsymbol{P} \\
\frac{\partial \boldsymbol{H}}{\partial \boldsymbol{B}}=\mu^{-1}, & \frac{\partial \eta}{\partial \boldsymbol{B}}=\boldsymbol{r}, & \frac{\partial \eta}{\partial \theta}=\alpha
\end{aligned}
$$

In Eq. (7) $\alpha$ is given as $\alpha=\rho c_{v} \Theta_{0}^{-1}$, where $c_{v}$ is specific heat. From Eqs. (3), (4), and (7)

$$
\begin{gathered}
\frac{\partial^{2} G}{\partial \boldsymbol{E} \partial \boldsymbol{B}}=-\frac{\partial \boldsymbol{D}}{\partial \boldsymbol{B}}=\frac{\partial \boldsymbol{H}}{\partial \boldsymbol{E}}=-b, \quad \frac{\partial^{2} G}{\partial \boldsymbol{E} \partial \theta}=-\frac{\partial \boldsymbol{D}}{\partial \theta}=-\frac{\partial \eta}{\partial \boldsymbol{E}}=-\boldsymbol{P} \\
\frac{\partial^{2} G}{\partial \boldsymbol{B} \partial \theta}=\frac{\partial \boldsymbol{H}}{\partial \theta}=-\frac{\partial \eta}{\partial \boldsymbol{B}}=-\boldsymbol{r}
\end{gathered}
$$

When Eqs. (5-8) are used in Eq. (4) under the assumption that in the natural state both the independent and dependent variables vanish, the following constitutive equations are obtained for a thermopiezomagnetic medium:

$$
\begin{gathered}
\boldsymbol{T}=\frac{\partial G}{\partial \boldsymbol{S}}=c \boldsymbol{S}-e \boldsymbol{E}-\ell \boldsymbol{B}-\lambda \theta \\
\boldsymbol{D}=-\frac{\partial G}{\partial \boldsymbol{E}}=e^{T} \boldsymbol{S}+\varepsilon \boldsymbol{E}+b^{T} \boldsymbol{B}+\boldsymbol{P} \theta \\
\boldsymbol{H}=\frac{\partial G}{\partial \boldsymbol{B}}=-\ell^{T} \boldsymbol{S}-b \boldsymbol{E}+\mu^{-1} \boldsymbol{B}-\boldsymbol{r} \theta \\
\eta=-\frac{\partial G}{\partial \theta}=\lambda^{T} \boldsymbol{S}+\boldsymbol{P}^{T} \boldsymbol{E}+\boldsymbol{r}^{T} \boldsymbol{B}+\alpha \theta
\end{gathered}
$$


It is easy to prove that the thermodynamic potential $G$ takes the following form:

$$
\begin{aligned}
G= & \frac{1}{2} \boldsymbol{S}^{T} c \boldsymbol{S}-\frac{1}{2} \boldsymbol{E}^{T} \varepsilon \boldsymbol{E}+\frac{1}{2} \boldsymbol{B}^{T} \mu^{-1} \boldsymbol{B}-\frac{1}{2} \alpha \theta^{2}-\boldsymbol{S}^{T} e \boldsymbol{E} \\
& -\boldsymbol{E}^{T} \boldsymbol{P} \theta-\boldsymbol{S}^{T} \lambda \theta-\boldsymbol{S}^{T} \ell \boldsymbol{B}-\boldsymbol{B}^{T} \boldsymbol{r} \theta-\boldsymbol{B}^{T} b \boldsymbol{E}
\end{aligned}
$$

\section{Differential Equations of Thermopiezomagnetism}

Two energy functionals $\Pi$ and $\Psi$ are defined as follows

$$
\begin{aligned}
\Pi= & \int_{V}(G+\eta \Theta) \mathrm{d} V-\int_{V} \boldsymbol{u}^{T} \boldsymbol{P}_{b} \mathrm{~d} V-\int_{S} \boldsymbol{u}^{T} \boldsymbol{P}_{s} \mathrm{~d} V+\int_{V} \phi \rho_{v} \mathrm{~d} V \\
& +\int_{S} \phi \sigma \mathrm{d} S-\int_{V} \boldsymbol{A}^{T} \boldsymbol{J} \mathrm{d} V+\int_{S} \boldsymbol{A}^{T} H_{E}^{\prime} \boldsymbol{d} S
\end{aligned}
$$$$
\Psi=\int_{V}(\Gamma-\mu \Theta \dot{\Theta}-W \Theta) \mathrm{d} V+\int_{S} \Theta \boldsymbol{h}^{T} \boldsymbol{n} \mathrm{d} S
$$

where $\Theta$ is given as $\Theta=\Theta_{0}+\theta$ and $H_{E}^{\prime}$ is the matrix of external magnetic field intensity defined in Cartesian coordinate system as

$$
H_{E}^{\prime}=\left[\begin{array}{ccc}
0 & H_{z} & -H_{y} \\
-H_{z} & 0 & H_{x} \\
H_{y} & -H_{x} & 0
\end{array}\right]_{E}
$$

$\Gamma$ in Eq. (11) is given as

$$
\Gamma=\frac{1}{2} \nabla^{T} \Theta K \nabla \Theta
$$

where $\nabla$ is the gradient vector. The generalizedHamilton's principle has the following forms:

$$
\begin{gathered}
\delta \int_{t_{1}}^{t_{2}}(K i-\Pi) \mathrm{d} t=0 \\
\delta \int_{t_{1}}^{t_{2}} \Psi \mathrm{d} t=0
\end{gathered}
$$

where the kinetic energy $K i$ is

$$
K i=\int_{V} \frac{1}{2} \rho \dot{\boldsymbol{u}}^{T} \dot{\boldsymbol{u}} \mathrm{d} t
$$

for which

$$
\delta \int_{t_{1}}^{t_{2}} K i \mathrm{~d} t=\delta \int_{t_{1}}^{t_{2}} \int_{V} \frac{1}{2} \rho \dot{\boldsymbol{u}}^{T} \dot{\boldsymbol{u}} \mathrm{d} t \mathrm{~d} V=-\int_{t_{1}}^{t_{2}} \mathrm{~d} t \int_{V} \rho \delta \boldsymbol{u}^{T} \ddot{\boldsymbol{u}} \mathrm{d} t
$$

Also, from Eqs. (2) and (3)

$$
\delta G=\delta \boldsymbol{S}^{T} \boldsymbol{T}-\delta \boldsymbol{E}^{T} \boldsymbol{D}+\delta \boldsymbol{B}^{T} \boldsymbol{H}-\delta \theta \eta
$$

Hence,

$$
\begin{aligned}
& \delta \int_{t_{1}}^{t_{2}}(K i-\Pi) \mathrm{d} t=\int_{t_{1}}^{t_{2}} \mathrm{~d} t\left[\int _ { V } \left(-\rho \delta \boldsymbol{u}^{T} \ddot{\boldsymbol{u}}-\delta \boldsymbol{S}^{T} \boldsymbol{T}\right.\right. \\
& \left.+\delta \boldsymbol{E}^{T} \boldsymbol{D}-\delta \boldsymbol{B}^{T} \boldsymbol{H}+\delta \boldsymbol{u}^{T} \boldsymbol{P}_{b}-\delta \phi^{T} \rho_{v}+\delta \boldsymbol{A}^{T} \boldsymbol{J}\right) \mathrm{d} V \\
& \left.\quad+\int_{S} \delta \boldsymbol{u}^{T} \boldsymbol{P}_{s} \mathrm{~d} S-\int_{S} \delta \phi^{T} \sigma \mathrm{d} S-\int_{S} \delta \boldsymbol{A}^{T} H_{E}^{\prime} \boldsymbol{n} \mathrm{d} S\right]=0
\end{aligned}
$$

Now recall the following relations:

$$
\boldsymbol{S}=L_{u} \boldsymbol{u}, \quad \boldsymbol{E}=-\nabla \phi-\dot{\boldsymbol{A}}, \quad \boldsymbol{B}=\nabla \times \boldsymbol{A}=L_{A} \boldsymbol{A}
$$

where $L_{u}$ and $L_{A}$ are differential operators. Substituting Eq. (20) into Eq. (19) yields

$$
\begin{array}{rl}
\int_{t_{1}}^{t_{2}} & \mathrm{~d} t\left\{\int _ { V } \left[-\rho \delta \boldsymbol{u}^{T} \ddot{\boldsymbol{u}}-\left(L_{u} \delta \boldsymbol{u}\right)^{\boldsymbol{T}} \boldsymbol{T}-(\nabla \delta \phi)^{T} \boldsymbol{D}-\delta \dot{\boldsymbol{A}}^{T} \boldsymbol{D}\right.\right. \\
& \left.-(\nabla \times \delta \boldsymbol{A})^{T} \boldsymbol{H}+\delta \boldsymbol{u}^{T} \boldsymbol{P}_{b}-\delta \phi^{T} \rho_{v}+\delta \boldsymbol{A}^{T} \boldsymbol{J}\right] \mathrm{d} V \\
& \left.+\int_{S} \delta \boldsymbol{u}^{T} \boldsymbol{P}_{S} \mathrm{~d} S-\int_{S} \delta \phi^{T} \sigma \mathrm{d} S-\int_{S} \delta \boldsymbol{A}^{T} H_{E}^{\prime} \boldsymbol{n} \mathrm{d} S\right\}=0
\end{array}
$$

The equalities for some terms in Eq. (21) can be written as

$$
\begin{aligned}
& \int_{t_{1}}^{t_{2}} \mathrm{~d} t \int_{V} \delta \dot{\boldsymbol{A}}^{T} \boldsymbol{D} \mathrm{d} V=-\int_{t_{1}}^{t_{2}} \mathrm{~d} t \int_{V} \delta \boldsymbol{A}^{T} \dot{\boldsymbol{D}} \mathrm{d} V \\
& \int_{V}\left(L_{u} \delta \boldsymbol{u}\right)^{T} \boldsymbol{T} \mathrm{d} V=-\int_{V} \delta \boldsymbol{u}^{T}\left(L_{u}^{T} \boldsymbol{T}\right) \mathrm{d} V+\int_{S} \delta \boldsymbol{u}^{T}(N \boldsymbol{T}) \mathrm{d} S \\
& \int_{V}(\nabla \delta \phi)^{T} \boldsymbol{D} \mathrm{d} V=\int_{V} \nabla^{T}(\boldsymbol{D} \delta \phi) \mathrm{d} V-\int_{V} \delta \phi^{T}\left(\nabla^{T} \boldsymbol{D}\right) \mathrm{d} V \\
& =\int_{S} \delta \phi^{T} \boldsymbol{D}^{T} \boldsymbol{n} \mathrm{d} S-\int_{V} \delta \phi^{T}\left(\nabla^{T} \boldsymbol{D}\right) \mathrm{d} V \\
& \int_{V}(\nabla \times \delta \boldsymbol{A})^{T} \boldsymbol{H} \mathrm{d} V=\int_{V} \nabla^{T}(\delta \boldsymbol{A} \times \boldsymbol{H}) \mathrm{d} V+\int_{V} \delta \boldsymbol{A}^{T}(\nabla \times \boldsymbol{H}) \mathrm{d} V
\end{aligned}
$$

where in the Cartesian coordinate system

$$
N=\left[\begin{array}{cccccc}
n_{x} & 0 & 0 & n_{y} & n_{z} & 0 \\
0 & n_{y} & 0 & n_{x} & 0 & n_{z} \\
0 & 0 & n_{z} & 0 & n_{x} & n_{y}
\end{array}\right], \quad \delta \boldsymbol{A} \times \boldsymbol{H}=H^{\prime} \delta \boldsymbol{A}
$$

with $n_{x}, n_{y}$, and $n_{z}$ being the Cartesian components of the surface normal $\boldsymbol{n}$ and $H^{\prime}$ being defined as in Eq. (12). Hence the fourth equality in Eq. (22) becomes

$$
\begin{gathered}
\int_{V}(\nabla \times \delta \boldsymbol{A})^{T} \boldsymbol{H} \mathrm{d} V=\int_{V} \nabla^{T}\left(H^{\prime} \delta \boldsymbol{A}\right) \mathrm{d} V+\int_{V} \delta \boldsymbol{A}^{T}(\nabla \times \boldsymbol{H}) \mathrm{d} V \\
=-\int_{S} \delta \boldsymbol{A}^{T} H^{\prime} \boldsymbol{n} \mathrm{d} S+\int_{V} \delta \boldsymbol{A}^{T}(\nabla \times \boldsymbol{H}) \mathrm{d} V
\end{gathered}
$$

Equation (21) is now transformed to

$$
\begin{array}{rl}
\int_{t_{1}}^{t_{2}} & \mathrm{~d} t\left[\int_{V} \delta \boldsymbol{u}^{T}\left(-\rho \ddot{\boldsymbol{u}}+L_{u}^{T} \boldsymbol{T}+\boldsymbol{P}_{b}\right) \mathrm{d} V\right. \\
& +\int_{V} \delta \phi^{T}\left(\nabla^{T} \boldsymbol{D}-\rho_{v}\right) \mathrm{d} V+\int_{V} \delta \boldsymbol{A}^{T}(\dot{\boldsymbol{D}}-\nabla \times \boldsymbol{H}+\boldsymbol{J}) \mathrm{d} V \\
& +\int_{S} \delta \boldsymbol{u}^{T}\left(\boldsymbol{P}_{s}-N \boldsymbol{T}\right) \mathrm{d} S-\int_{S} \delta \phi^{T}\left(\sigma+\boldsymbol{D}^{T} \boldsymbol{n}\right) \mathrm{d} S \\
& \left.-\int_{S} \delta \boldsymbol{A}^{T}\left(H_{E}^{\prime}-H^{\prime}\right) \boldsymbol{n} \mathrm{d} S\right]=0
\end{array}
$$

Therefore, the following equations must be satisfied with appropriate boundary conditions on the corresponding surfaces:

$$
\begin{gathered}
-\rho \ddot{\boldsymbol{u}}+L_{u}^{T} \boldsymbol{T}+\boldsymbol{P}_{b}=0, \quad \nabla^{T} \boldsymbol{D}-\rho_{v}=0 \\
\dot{\boldsymbol{D}}-\nabla \times \boldsymbol{H}+\boldsymbol{J}=0
\end{gathered}
$$

The first equation in Eq. (26) is the equation of motion for the mechanical field, and the second and third equations are the Maxwell's equilibrium equations for the electrical and magnetic fields. ${ }^{20}$ 
It can be shown ${ }^{9}$ that the Hamilton's principle on $\Psi$ given in Eq. (15) yields

$$
\int_{t_{1}}^{t_{2}} \mathrm{~d} t\left[\int_{V} \delta \theta\left(\nabla^{T} \boldsymbol{q}-W+\Theta \dot{\eta}\right) \mathrm{d} V+\int_{S} \delta \theta\left(\boldsymbol{h}^{T}-\boldsymbol{q}^{T}\right) \boldsymbol{n} \mathrm{d} S\right]=0
$$

Hence the principle yields the generalized heat equation given as

$$
\nabla^{T} \boldsymbol{q}-W+\Theta \dot{\eta}=0
$$

with the boundary condition on the surface being $\boldsymbol{q}=\boldsymbol{h}$.

\section{Finite Element Equations for a Thermopiezomagnetic Medium}

The variables of the finite element formulation are chosen as $\boldsymbol{u}$, $\phi, \boldsymbol{A}$, and $\theta$ for the mechanical, electrical, magnetic, and thermal fields, respectively. The following approximations are written for each finite element:

$$
\boldsymbol{u}_{e}=N_{u} \boldsymbol{u}_{i}, \quad \phi_{e}=N_{\phi} \phi_{i}, \quad \boldsymbol{A}_{e}=N_{A} \boldsymbol{A}_{i}, \quad \theta_{e}=N_{\theta} \theta_{i}
$$

where the subscripts $e$ and $i$ respectively stand for the element and nodes of the element and $N$ are the shape function matrices whose subscripts denote the associated fields. The following relations are also written:

$$
\begin{gathered}
\boldsymbol{S}_{e}=L_{u} \boldsymbol{u}_{e}=\left[L_{u} N_{u}\right] \boldsymbol{u}_{i}=B_{u} \boldsymbol{u}_{i} \\
\boldsymbol{E}_{e}=-\nabla \phi_{e}-\dot{\boldsymbol{A}_{e}}=-\left[\nabla N_{\phi}\right] \phi_{i}-N_{A} \dot{\boldsymbol{A}}_{i}=-B_{\phi} \phi_{i}-N_{A} \dot{\boldsymbol{A}}_{i} \\
\boldsymbol{B}_{e}=L_{A} \boldsymbol{A}_{e}=\left[L_{A} N_{A}\right] \boldsymbol{A}_{i}=B_{A} \boldsymbol{A}_{i} \\
\nabla \theta_{e}=\left[\nabla N_{\theta}\right] \theta_{i}=B_{\theta} \theta_{i}
\end{gathered}
$$

SubstitutingEqs. (9), (29), and (30) in Eqs. (15) and (21) resultsin the following coupled finite element equations (after assemblage):

$$
\begin{aligned}
& M_{u u} \ddot{\boldsymbol{u}}+C_{u A} \dot{\boldsymbol{A}}+K_{u u} \boldsymbol{u}+K_{u \phi} \phi-K_{u A} \boldsymbol{A}-K_{u \theta} \theta=\boldsymbol{F} \\
& -C_{\phi A} \dot{\boldsymbol{A}}+K_{\phi u} \boldsymbol{u}-K_{\phi \phi} \phi+K_{\phi A} \boldsymbol{A}+K_{\phi \theta} \theta=\boldsymbol{G} \\
& M_{A A} \ddot{\boldsymbol{A}}-C_{A u} \dot{\boldsymbol{u}}+C_{A \phi} \dot{\phi}+C_{A A} \dot{\boldsymbol{A}}-C_{A \theta} \dot{\theta} \\
& \quad-K_{A u} \boldsymbol{u}+K_{A \phi} \phi+K_{A A} \boldsymbol{A}-K_{A \theta} \theta=\boldsymbol{M} \\
& M_{\theta A} \ddot{\boldsymbol{A}}-C_{\theta u} \dot{\boldsymbol{u}}+C_{\theta \phi} \dot{\phi}-C_{\theta A} \dot{\boldsymbol{A}}-C_{\theta \theta} \dot{\theta}-K_{\theta \theta} \theta=\boldsymbol{Q}
\end{aligned}
$$

The element matrices in Eq. (31) are found as

$$
\begin{array}{rlrl}
{\left[M_{u u}\right]_{e}} & =\int_{V_{e}} \rho_{e} N_{u}^{T} N_{u} \mathrm{~d} V, & {\left[C_{u A}\right]_{e}} & =\int_{V_{e}} B_{u}^{T} e_{e} N_{A} \mathrm{~d} V \\
{\left[K_{u u}\right]_{e}} & =\int_{V_{e}} B_{u}^{T} c_{e} B_{u} \mathrm{~d} V, & {\left[K_{u \phi}\right]_{e}} & =\int_{V_{e}} B_{u}^{T} e_{e} B_{\phi} \mathrm{d} V \\
{\left[K_{u A}\right]_{e}} & =\int_{V_{e}} B_{u}^{T} \ell_{e} B_{A} \mathrm{~d} V, & {\left[K_{u \theta}\right]_{e}=\int_{V_{e}} B_{u}^{T} \lambda_{e} N_{\theta} \mathrm{d} V} \\
{\left[C_{\phi A}\right]_{e}=} & \int_{V_{e}} B_{\phi}^{T} \varepsilon_{e} N_{A} \mathrm{~d} V, & {\left[K_{\phi \phi}\right]_{e}} & =\int_{V_{e}} B_{\phi}^{T} \varepsilon_{e} B_{\phi} \mathrm{d} V \\
{\left[K_{\phi A}\right]_{e}} & =\int_{V_{e}} B_{\phi}^{T} b_{e}^{T} B_{A} \mathrm{~d} V, & {\left[K_{\phi \theta}\right]_{e}} & =\int_{V_{e}} B_{\phi}^{T} P_{e} N_{\theta} \mathrm{d} V \\
& {\left[C_{A A}\right]_{e}=\int_{V_{e}}\left(B_{A}^{T} b_{e} N_{A}-N_{A}^{T} b_{e}^{T} B_{A}\right) \mathrm{d} V} \\
{\left[M_{A A}\right]_{e}=} & \int_{V_{e}} N_{A}^{T} \varepsilon_{e} N_{A} \mathrm{~d} V, & {\left[C_{A \theta}\right]_{e}=\int_{V_{e}} N_{A}^{T} P_{e} N_{\theta} \mathrm{d} V} \\
{\left[K_{A A}\right]_{e}=} & \int_{V_{e}} B_{A}^{T} \mu_{e}^{-1} B_{A} \mathrm{~d} V, & {\left[K_{A \theta}\right]_{e}=\int_{V_{e}} B_{A}^{T} r_{e} N_{\theta} \mathrm{d} V}
\end{array}
$$

$$
\begin{aligned}
{\left[M_{\theta A}\right]_{e}=\int_{V_{e}} \Theta_{o e} N_{\theta}^{T} P_{e}^{T} N_{A} \mathrm{~d} V, } & {\left[C_{\theta u}\right]_{e}=\int_{V_{e}} \Theta_{o e} N_{\theta}^{T} \lambda_{e}^{T} B_{u} \mathrm{~d} V } \\
{\left[C_{\theta \phi}\right]_{e}=\int_{V_{e}} \Theta_{o e} N_{\theta}^{T} P_{e}^{T} B_{\phi} \mathrm{d} V, } & {\left[C_{\theta A}\right]_{e}=\int_{V_{e}} \Theta_{o e} N_{\theta}^{T} r_{e}^{T} B_{A} \mathrm{~d} V } \\
{\left[C_{\theta \theta}\right]_{e}=\int_{V_{e}} \Theta_{o e} N_{\theta}^{T} \alpha_{e} N_{\theta} \mathrm{d} V, } & {\left[K_{\theta \theta}\right]_{e}=\int_{V_{e}} B_{\theta}^{T} K_{e} B_{\theta} \mathrm{d} V }
\end{aligned}
$$

In Eq. (31), $K_{\phi u}=K_{u \phi}^{T}, K_{A u}=K_{u A}^{T}, K_{A \phi}=K_{\phi A}^{T}, C_{A u}=C_{u A}^{T}$, and $C_{A \phi}=C_{\phi A}^{T}$. The elemental applied mechanical force, electrical charge, magnetic current, and heat vectors $(\boldsymbol{F}, \boldsymbol{G}, \boldsymbol{M}$, and $\boldsymbol{Q})$ in Eq. (31) are given as

$$
\begin{gathered}
\boldsymbol{F}_{e}=\int_{V_{e}} N_{u}^{T} \boldsymbol{P}_{\mathrm{be}} \mathrm{d} V+\int_{S_{e}} N_{u}^{T} \boldsymbol{P}_{\mathrm{se}} \mathrm{d} S+N_{u}^{T} \boldsymbol{P}_{\mathrm{ce}} \\
\boldsymbol{G}_{e}=-\int_{V_{e}} N_{\phi}^{T} \rho_{\mathrm{ve}} \mathrm{d} V-\int_{S_{e}} N_{\phi}^{T} \sigma_{e} \mathrm{~d} S \\
\boldsymbol{M}_{e}=\int_{V_{e}} N_{A}^{T} \boldsymbol{J}_{e} \mathrm{~d} V-\int_{S_{e}} B_{A}^{T} H_{\mathrm{Ae}}^{\prime} \boldsymbol{n} \mathrm{d} S \\
\boldsymbol{Q}_{e}=-\int_{V_{e}} N_{\theta}^{T} W_{e} \mathrm{~d} V+\int_{S_{e}} N_{\theta}^{T} \boldsymbol{h}_{e}^{T} \boldsymbol{n} \mathrm{d} S
\end{gathered}
$$

where for $\boldsymbol{F}_{e}$ the concentratedforce $\boldsymbol{P}_{\text {ce }}$ is also added. Equation (31) can be written in collective form as

$$
\begin{gathered}
{\left[\begin{array}{cccc}
M_{u и} & 0 & 0 & 0 \\
0 & 0 & 0 & 0 \\
0 & 0 & M_{A A} & 0 \\
0 & 0 & M_{\theta A} & 0
\end{array}\right]\left\{\begin{array}{c}
\ddot{u} \\
\ddot{\phi} \\
\ddot{A} \\
\ddot{\theta}
\end{array}\right\}} \\
+\left[\begin{array}{cccc}
0 & 0 & C_{u A} & 0 \\
0 & 0 & -C_{\phi A} & 0 \\
-C_{A u} & C_{A \phi} & C_{A A} & -C_{A \theta} \\
-C_{\theta u} & C_{\theta \phi} & -C_{\theta A} & -C_{\theta \theta}
\end{array}\right]\left\{\begin{array}{c}
\dot{\boldsymbol{u}} \\
\dot{\phi} \\
\dot{\boldsymbol{A}} \\
\dot{\theta}
\end{array}\right\} \\
+\left[\begin{array}{cccc}
K_{u u} & K_{u \phi} & -K_{u A} & -K_{u \theta} \\
K_{\phi u} & -K_{\phi \phi} & K_{\phi A} & K_{\phi \theta} \\
-K_{A u} & K_{A \phi} & K_{A A} & -K_{A \theta} \\
0 & 0 & 0 & -K_{\theta \theta}
\end{array}\right]\left\{\begin{array}{c}
\boldsymbol{u} \\
\phi \\
\boldsymbol{A} \\
\theta
\end{array}\right\}=\left\{\begin{array}{c}
\boldsymbol{F} \\
\boldsymbol{G} \\
\boldsymbol{M} \\
\boldsymbol{Q}
\end{array}\right\}
\end{gathered}
$$

Equation (31) or (34) can be used to investigate the dynamical behavior of a thermopiezomagnetic material where the mechanical, electrical, magnetic, and thermal fields are coupled through variables of $\boldsymbol{u}, \phi, \boldsymbol{A}$, and $\theta$.

\section{Numerical Example}

A composite beam shown in Fig. 1 is taken as an example to illustrate the use of thermopizeomagnetic equations given in preceding sections. The beam is composed of two bonded layers. The top layer is made up of a magnetostrictive ceramic, $\mathrm{CoFe}_{2} \mathrm{O}_{4}$, and the bottom layer is a piezoelectric ceramic, $\mathrm{BaTiO}_{3}$. Material properties of these materials are assumed as in Table 1 (Refs. 15 and 16). The dimensions of the beam are taken as follows: length $L=0.1 \mathrm{~m}$, height $h=0.001 \mathrm{~m}$, and width $b=0.005 \mathrm{~m}$.

As shown in Fig. 1, an electrical field is generated in the piezoelectric layer by applying an electrical voltage $-V$ to its bottom surface. The magnitude of $V$ is taken as unity (1 V).

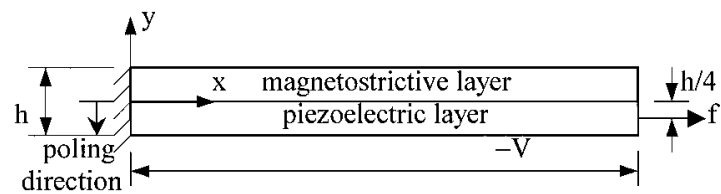

Fig. 1 Thermopiezomagnetic beam. 
Table 1 Properties of materials

\begin{tabular}{|c|c|}
\hline Property & Value \\
\hline \multicolumn{2}{|c|}{ Piezoceramic $\left(\mathrm{BaTiO}_{3}\right)$} \\
\hline$c_{11}, \mathrm{~Pa}$ & $1.3793 \times 10^{11}$ \\
\hline$e_{31}, \mathrm{C} / \mathrm{m}^{2}$ & 10.76 \\
\hline$\varepsilon_{11}, \varepsilon_{33}, \mathrm{~F} / \mathrm{m}$ & $1.19 \times 10^{-8}$ \\
\hline \multicolumn{2}{|c|}{ Magnetoceramic $\left(\mathrm{CoFe}_{2} \mathrm{O}_{4}\right)$} \\
\hline$c_{11}, \mathrm{~Pa}$ & $1.5432 \times 10^{11}$ \\
\hline$\ell_{31}, \mathrm{~N} / \mathrm{Wb}$ or $\mathrm{A} / \mathrm{m}$ & $2.86 \times 10^{8}$ \\
\hline$\mu_{11}, \mu_{33}, \mathrm{H} / \mathrm{m}$ & $6 \pi \times 10^{-8}$ \\
\hline
\end{tabular}

\section{Analytical Method}

A normal stress in the $x$ or 1 direction is induced in the beam as a result of the applied voltage. This normal stress is because an equivalent force of magnitude

$$
f=e_{31} E_{3} b h / 2=e_{31} V b
$$

is generated in the $x$ direction as shown in Fig. 1. In Eq. (35) a uniform electrical field of $E_{3}=2 \mathrm{~V} / \mathrm{h}$ is assumed across the piezoceramic layer. This force generates a bending moment at the neutral axis of the composite beam whose location is calculated using the transformed-section method. ${ }^{21}$ Here, the composite beam is transformed to a beam made up of the magnetostrictive material. The bending moment $m$ caused by $f$ is found as

$$
m=f(\bar{y}-h / 4)
$$

where $\bar{y}$ is the distance of the neutral axis from the beam's bottom surface. This moment brings about the vertical displacement $u_{3}$ in the beam given as

$$
u_{3}=\left(m / 2 Y_{m} I\right) x^{2}
$$

where $Y_{m}$ is the Young's modulus for the magnetostrictive material.

Normal stresses $T_{b}$ and $T_{t}$ at the bottom and top surfaces of the magnetostrictive layer of the beam are easily computed as

$$
T_{b}=(m / I) y_{b}+f / A, \quad T_{t}=-(m / I) y_{t}+f / A
$$

where $y_{b}$ and $y_{t}$ indicate the distances of the bottom and top surfaces of the magnetostrictivelayer from the neutral axis and $A$ is the crosssectional area of the transformed section.

After finding the normal stresses, the strains $S_{11}$ are calculated using the Hooke's law. The vertical component of the magnetic field $\mathrm{H}_{3}$ in the magnetoceramic layer is then found by the constitutive relation

$$
H_{3}=-\ell_{31} S_{11}
$$

\section{Finite Element Method}

For the finite element modeling and analysis each layer of the composite beam is divided into five equal elements, totaling 10 elements for the whole beam (Fig. 2). The displacement $\boldsymbol{u}$ of the beam can be found from the static finite element equations of the piezoelectric layer given as

$$
K_{u u} \boldsymbol{u}+K_{u \phi} \phi=\mathbf{0}, \quad K_{\phi u} \boldsymbol{u}-K_{\phi \phi} \phi=\boldsymbol{G}
$$

Once the displacement $\boldsymbol{u}$ of the whole beam through the finite element method is found, the strain $\boldsymbol{S}_{e}$ for each element can be computed using

$$
\boldsymbol{S}_{e}=B_{u} \boldsymbol{u}_{i}
$$

which is also indicated by Eq. (30). Then $\mathrm{H}_{3}$ for each element of the magnetoceramic layer can be computed via Eq. (39). Or, alternatively, the magnetic potential $\boldsymbol{A}$ for the the magnetoceramic layer can be found from Eq. (31) as

$$
\boldsymbol{A}=K_{A A}^{-1} K_{A u} \boldsymbol{u}
$$

Table 2 Magnetic field $H_{3}$ in $A / m$ for magnetostrictive layer

\begin{tabular}{lrr}
\hline \hline Surface & $\begin{array}{c}\text { Analytical } \\
\text { method }\end{array}$ & $\begin{array}{r}\text { Finite element } \\
\text { method }\end{array}$ \\
\hline Top & 11.44 & 11.42 \\
Bottom & -21.99 & -21.88 \\
\hline \hline
\end{tabular}

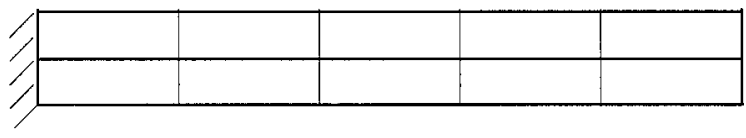

Fig. 2 Finite element mesh for thermopiezomagnetic beam.

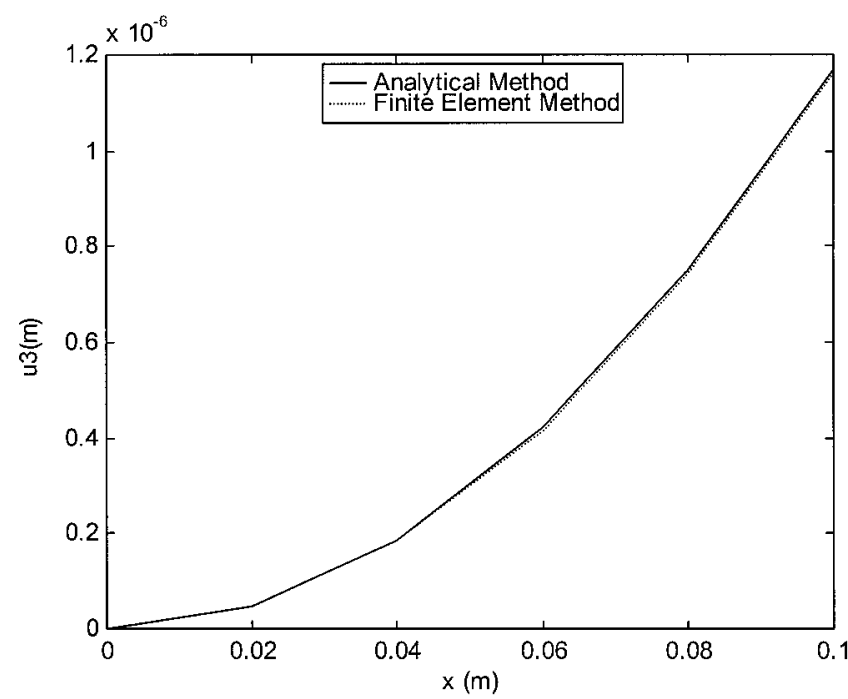

Fig. 3 Vertical displacement of beam subjected to vertical electrical field.

from which the magnetic field $\boldsymbol{H}$ for each element can be calculated as

$$
\boldsymbol{H}_{e}=\mu_{e}^{-1} B_{A} \boldsymbol{A}_{i}
$$

Vertical displacements $u_{3}$ of the beam computed by the analytical and finite element methods at different locations $x$ are plotted in Fig. 3. This figure illustrates the close agreement between the analytical and finite element results for $u_{3}$.

Magnetic fields in the $y$ direction $\mathrm{H}_{3}$ for the bottom and top surfaces of the magnetoceramic layer are calculated using the analytical and finite element methods. The results are tabulated in Table 2. There is a good match between the two methods.

\section{Conclusions}

The constitutive equations of thermopiezomagnetism are obtained through a thermodynamic potential. These expressions are the linear relations for a thermopiezomagnetic medium where mechanical, electrical, magnetic, and thermal fields interact with each other. Two energy functionals are used in Hamilton's principle, and the finite element method is employed to obtain finite element equations describing the dynamic behavior of the thermopiezomagnetic medium.

A thermopiezomagnetic composite beam is formed by bonding together layers of piezoelectric and magnetostrictive ceramics. The piezoelectric layer is subjected to an electrical field, which causes normal strains in the beam. The strains in the magnetostrictivelayer then lead to the generation of magnetic field. Analytical and finite element solutions are found for the vertical components of the displacement $u_{3}$ and magnetic field $\mathrm{H}_{3}$. The results indicate a close agreement between analytical and finite element solutions.

This paper presents a variational approach of obtaining general coupled-field equations for thermopiezomagnetic composites. These composites can be used as sensors and actuators that are 
essential elements for smart/intelligent structures such as those found in aerospace applications. Hence, the thermopiezomagnetic equations derived here are expected to be important in describing their behavior.

\section{Acknowledgment}

The authors gratefully acknowledge the support of King Fahd University of Petroleum and Minerals in carrying out this research.

\section{References} 295.

${ }^{1}$ Curie, J., and Curie, P., Comptes Rendus, Vol. 91, Paris, 1880, pp. 294

${ }^{2}$ Tzou, H. S., and Guran, A., (eds.), Structronic Systems: Smart Structures, Devices and System, Part 2: Systems and Control, World Scientific, River Edge, NJ, 1998.

${ }^{3}$ Callahan, J., and Baruh, H., "Modal Sensing of Cylindrical Shells Using Segmented Piezoelectric Elements," Smart Materials and Structures, Vol. 8 , No. 1, 1999, pp. 125-135.

${ }^{4}$ Crawley, E. F., "Intelligent Structures for Aerospace: A Technology Overview and Assessment," Journal of Aircraft, Vol. 32, No. 8, 1994 , pp. 1689-1699.

${ }^{5}$ Newnham, R. E., "Composite Sensors and Actuators," Society of PhotoOptical Instrumentation Engineers Conference on Smart Structures and Materials, Vol. 2716, Society of Photo-Optical Instrumentation Engineers, Bellingham, WA, 1996, pp. 347-349.

${ }^{6}$ Sunar, M., and Rao, S. S., "Recent Advances in Sensing and Control of Flexible Structures via Piezoelectric Materials Technology," Applied Mechanics Reviews, Vol. 52, No. 1, 1999, pp. 1-16.

${ }^{7}$ Mindlin, R. D., "On the Equations of Motion of Piezoelectric Crystals," Problems of Continuum Mechanics, edited by J. Radok, Society for Industrial and Applied Mechanics, Philadelphia, 1961, pp. 282-290.

${ }^{8}$ Mindlin, R. D., "Equations of High Frequency Vibrations of Thermopiezoelectric Crystal Plates," International Journal of Solids and Structures, Vol. 10, No. 6, 1974, pp. 625-637.

${ }^{9}$ Nowacki, W., "Some General Theorems of Thermopiezoelectricity," Journal of Thermal Stresses, Vol. 1, No. 2, 1978, pp. 171-182.
${ }^{10}$ Rao, S. S., and Sunar, M., "Analysis of Distributed Thermopiezoelectric Sensors and Actuators in Advanced Intelligent Structures," AIAA Journal, Vol. 31, No. 7, 1993, pp. 1280-1286.

${ }^{11}$ Sunar, M., and Rao, S. S., "Thermopiezoelectric Control Design and Actuator Placement," AIAA Journal, Vol. 35, No. 3, 1997, pp. 534-539.

${ }^{12}$ Tzou, H. S., and Ye, R., "Piezothermoelasticity and Precision Control of Piezoelectric Systems: Theory and Finite Element Analysis," Journal of Vibration and Acoustics, Vol. 116, No. 4, 1994, pp. 489-495.

${ }^{13}$ Tauchert, T. R., "Piezothermoelastic Behavior of a Laminated Plate," Journal of Thermal Stresses, Vol. 15, No. 1, 1992, pp. 25-37.

${ }^{14}$ Body, C., Reyne, G., and Meunier, G., "Modeling of Magnetostrictive Thin Films, Application to a Micromembrane," Journal of Physics III France, Vol. 7, No. 1, 1997, pp. 67-85.

${ }^{15}$ Harshe, G., Dougherty, J. P., and Newnham, R. E., "Theoretical Modeling of Multilayer Magnetoelectric Composites," International Journal of Applied Electromagnetics in Materials, Vol. 4, No. 2, 1993, pp. 145-159.

${ }^{16}$ Avellaneda, M., and Harshe, G., "Magnetoelectric Effect in Piezoelectric/Magnetostrictive Multilayer (2-2) Composites," Journal of Intelligent Material Systems and Structures, Vol. 5, No. 4, 1994, pp. 501513.

${ }^{17}$ Arai, K. I., Muranaka, C. S., and Yamaguchi, M., "A New Hybrid Device Using Magnetostrictive Amorphous Films and Piezoelectric Substrates," IEEE Transactions on Magnetics, Vol. 30, No. 2, 1994, pp. 916918

${ }^{18}$ Prieto, J. L., Aroca, C., Sanchez, M. C., Lopez, E., and Sanchez, P., "Magnetization Processes and Optimal Performance of Magnetostrictive Piezoelectric Sensors," Journal of Applied Physics, Vol. 79, No. 9, 1996, pp. 7099-7105.

${ }^{19}$ Nowacki, W., Dynamic Problems of Thermoelasticity, Noordhoff International, Leiden, the Netherlands, 1975, pp. 327, 328

${ }^{20}$ Sadiku, M. N. O., Elements of Electromagnetics, 2nd ed., Oxford Univ. Press, New York, 1995, p. 428.

${ }^{21}$ Hibbeler, R. C., Mechanics of Materials, 3rd ed., Prentice-Hall, Upper Saddle River, NJ, 1997, pp. 319-325.

A. M. Baz Associate Editor 